University of Nebraska - Lincoln

DigitalCommons@University of Nebraska - Lincoln

Mechanical \& Materials Engineering Faculty

Publications

Mechanical \& Materials Engineering,

Department of

$1-15-2009$

\title{
Analysis of Flux-Base Fins for Estimation of Heat Transfer Coefficient
}

Kevin D. Cole

University of Nebraska-Lincoln, kcole1@unl.edu

C. Tarawneh

University of Texas-Pan American

B. Wilson

University of Texas-Pan American

Follow this and additional works at: https://digitalcommons.unl.edu/mechengfacpub

Part of the Mechanical Engineering Commons

Cole, Kevin D.; Tarawneh, C.; and Wilson, B., "Analysis of Flux-Base Fins for Estimation of Heat Transfer Coefficient" (2009). Mechanical \& Materials Engineering Faculty Publications. 25.

https://digitalcommons.unl.edu/mechengfacpub/25

This Article is brought to you for free and open access by the Mechanical \& Materials Engineering, Department of at DigitalCommons@University of Nebraska - Lincoln. It has been accepted for inclusion in Mechanical \& Materials Engineering Faculty Publications by an authorized administrator of DigitalCommons@University of Nebraska Lincoln. 
Published in International Journal of Heat and Mass Transfer 52:1-2 (January 15, 2009), pp. 92-99;

doi: 10.1016/j.ijheatmasstransfer.2008.05.031 Copyright (C) 2008 Elsevier Ltd. Used by permission. http://www.elsevier.com/locate/ijhmt

Submitted March 3, 2008; revised May 31, 2008; published online July 27, 2008.

\title{
Analysis of flux-base fins for estimation of heat transfer coefficient
}

\author{
K. D. Cole, ${ }^{\mathrm{a}}$ C. Tarawneh, ${ }^{\mathrm{b}}$ and B. Wilson ${ }^{\mathrm{a}}$ \\ a University of Nebraska-Lincoln, Department of Mechanical Engineering, \\ N104 Walter Scott Engineering Center, Lincoln, NE 68588-0656, USA \\ ${ }^{\mathrm{b}}$ University of Texas-Pan American, Department of Mechanical Engineering, \\ 1201 West University Drive, Edinburg, TX 78539, USA \\ Corresponding author - K. D. Cole, tel 402 472-5857, fax 402 472-1465, email kcole1@unl.edu
}

\begin{abstract}
Exact solutions are given for the transient temperature in flux-base fins with the method of Green's functions (GF) in the form of infinite series for three different tip conditions. The speed of convergence is improved by replacing the steady part by a closed-form steady solution. For the insulated-tip case, a quasi-steady solution is presented. Numerical values are presented and the conditions under which the quasi-steady solution is accurate are determined. An experimental example is given for estimation of the heat transfer coefficient (HTC) on a non-rotating roller bearing, in which the outer bearing race is treated as a transient fin.
\end{abstract}

Keywords: transient conduction, heat transfer coefficient, parameter estimation, extended surface, Neumann boundary

\section{Introduction}

The transient response of fins is important in a wide range of engineering devices including heat exchangers, clutches, motors and so on. The authors' interest in this topic grew out of analysis of transient data for determination of surface heat transfer coefficients on railroad roller bearings, in which the outer race of the bearing is treated as a fin. Next a review of literature is given in the areas of transient fins and in transient experiments for estimation of heat transfer coefficients.

There are several papers on transient heat transfer in fins, starting with Chapman [1] who studied the transient behavior of an annular fin of uniform thickness subjected to a sudden step change in the base temperature. His interest in circular annular fins stemmed from the numerous applications of these types of fins, especially on cylinders of air-cooled internal combustion engines. Chapman developed equations that give the temperature distribution within the fin, the heat removed from the source, and the heat dissipated to the surroundings, all as functions of time. He also presents his equations in graphical form for the use of design engineers. Donaldson and Shouman [2] studied the transient temperature distribution in a convecting straight fin of constant area for two distinct cases, namely, a step change in base temperature, and a step change in base heat flow rate. The tip of the fin is insulated. The authors developed the equations for the transient temperature distribution and the heat flow rate for the two aforementioned cases, and present their results graphically. Also included is a summary of their experimental work to verify their results for the case of a step function in heat flow rate. In a series of papers, Suryanarayana $[3,4]$ also studied the transient response of straight fins of constant cross-sectional area. However, rather than using the separation of variables technique followed by Donaldson and Shouman, he utilized the Laplace transforms in order to develop the solutions for small and large values of time when the base of the fin is subjected to a step change in temperature or heat flux. The tip of the fin is insulated. In addition, the use of the Laplace transforms made it easier for Suryanarayana to develop solutions for the case of a fin subjected to a sinusoidal temperature or heat flux at its base. His second paper on the subject provided an analysis of the heat transfer that takes place from one fluid to another separated by a solid boundary with fins on one side. Mao and Rooke [5] also used the Laplace transform method to study straight fins with three different transients: a step change in base temperature; a step change in base heat flux; and, a step change in fluid temperature.

Transient fins of constant cross-section have also been studied with the method of Green's functions [6, pp. 60-64], a flexible and powerful approach that are applicable to any combination of end conditions on the fin. Kim [7] developed an approximate solution to the transient heat transfer in straight fins of constant cross-sectional area and constant physical and thermal properties. The author utilized the Kantorovich method in the variational formulation to provide a simpler expression of the exact form of the solution.

In some fin applications, Newton's law of cooling is not applicable, and a power-law type dependence of convective heat flux on temperature better describes the cooling process. Such cases in- 


\begin{tabular}{|llll|}
\hline \multicolumn{2}{|l|}{ Nomenclature } & $V$ & fin volume $\left(\mathrm{m}^{3}\right)$ \\
$A_{h}$ & surface area of fin for convection $\left(\mathrm{m}^{2}\right)$ & $W$ & transformed temperature, Equation $(25)$ \\
$B i$ & Biot number, $h_{i}\left(V / A_{h}\right) / k$ & Greek & \\
$B_{2}$ & Biot number, $h \mathrm{~L} / k$ & & \\
$\mathrm{G}$ & Green's function & $\alpha$ & thermal diffusivity $\left(\mathrm{m}^{2} \mathrm{~s}^{-1}\right)$ \\
$h$ & heat transfer coefficient $\left(\mathrm{W} \mathrm{m}^{-2} \mathrm{~K}^{-1}\right)$ & $\beta_{n}$ & $\begin{array}{l}\text { eigenvalue, Equation }(31) \\
\text { cylindrical shell thickness, } \mathrm{m}\end{array}$ \\
$k$ & thermal conductivity $\left(\mathrm{W} \mathrm{m}^{-1} \mathrm{~K}^{-1}\right)$ & $\delta$ & dimensionless temperature \\
$L$ & length of fin $(\mathrm{m})$ & $\theta$ & dimensionless $x$-coordinate \\
$N_{n}$ & norm, Equation $(31)(\mathrm{m})$ & $\xi$ & dimensionless time \\
$m$ & fin parameter, Equation $(1),\left(\mathrm{m}^{-1}\right)$ & $\tau$ & \\
$M$ & dimensionless fin parameter $=\mathrm{mL}^{-2}$ & Superscripts \\
$q_{o}$ & heat flux $(\mathrm{W} \mathrm{m})$ & $L$ & lumped capacitance \\
$Q$ & input heat $(\mathrm{W})$ & $q$ & quasi-steady \\
$T$ & temperature $(\mathrm{K})$ & $s$ & steady-state \\
$t$ & time $(\mathrm{s})$ & & \\
& & & \\
& & & \\
\hline
\end{tabular}

clude cooling of fins due to film boiling, natural convection, nucleate boiling, and radiation to space at absolute zero. Aziz and Na [8] considered the transient response of a semi-infinite fin of uniform thickness, initially at the ambient temperature, subjected to a step change in temperature at its base, with fin cooling governed by a power-law type dependence on temperature difference. The choice of a semi-infinite geometry enabled the transformation of the governing nonlinear partial differential equations into a sequence of similarity type linear perturbation equations. Aziz and $\mathrm{Na}$ also discussed the applicability of the results to finite fins.

Aziz and Kraus [9] present a variety of analytical results for transient fins, developed by separation of variable and Laplace transform techniques. Results discussed include rectangular fins with three different base conditions, rectangular fins with power-law convective heat loss, and radial fins, along with several specific examples. Aziz and Kraus also present a comprehensive literature review. The material on transient fins of constant cross-section is also included in a book by Kraus, Aziz and Welty [10, ch. 16].

The work discussed so far has focused on the transient response of fins of simple geometry such as circular annular fins and straight fins. In addition, several simplifying assumptions were utilized such as uniform thickness, constant cross-sectional area, semi-infinite length, insulated tip, and small fin thickness-tolength ratio to ensure one-dimensional heat conduction. Recently, work has included fins of various shapes and cross-sections, twoand three-dimensional heat transfer, and practical applications of finned heat exchangers. Tseng et al. [11] analyzed the transient heat transfer in two-dimensional straight fins of various shapes subjected at their base to a decayed exponential function of time in heat flux. The latter authors used Laplace transforms and integral methods to obtain solutions. The same solution technique was also utilized by Cheng and Chen [12] to study the transient response of annular fins of various shapes exposed to specified heat flux at the base. The shapes studied were fins with rectangular, triangular, and parabolic profiles. Approximate treatment of two-dimensional heat conduction in short rectangular fins was carried out by Ju et al. [13] with a perturbation technique, by Onur [14] with an averaging technique, and by Singh [15] by a variational method. These studies account for cross-axis heat conduction in short fins.

Campo and Salazar [16] explored the analogy between the transient conduction in a planar slab for short times and the steadystate conduction in a straight fin of uniform cross-section. They made use of a hybrid computational method, known as the Transversal Method Of Lines (TMOL), to arrive at approximate analytical solutions of the unsteady-state heat conduction equation for short times in a plane having a uniform initial temperature and subjected to a Dirichlet boundary condition. The resulting solutions are suitable for obtaining quality short-time temperature distributions within the slab when it is subjected to a Dirichlet boundary condition, or a Robin boundary condition for which the convective heat transfer coefficient is very large and/or the thermal conductivity of the slab material is very small. In an application type study, Saha and Acharya [17] conducted a detailed parametric analysis of the unsteady three-dimensional flow and heat transfer in a pin-fin heat exchanger. The work was motivated by the desire to enhance the performance of compact heat exchangers, which are designed to provide high heat transfer surface area per unit volume and to alter the fluid dynamics to enhance mixing. There have been several numerical studies of transient fins combined with complicating factors, such as natural convection $[18,19]$, spatial arrays of fins [20-22], and phase change materials [23].

There are few publications on transient experiments for determining heat transfer coefficients in fins. Mutlu and Al-Shemmeri [24] studied a longitudinal array of straight fins suddenly heated at the base. The instantaneous heat transfer coefficient was found at one point on the fin as a ratio of the measured temperature to the measured heat flux. There are several papers on inverse techniques for determination of heat transfer coefficient from temperatures measured in compact bodies suddenly placed in a convection environment [25-29]. In these studies, the heat transfer coefficient is found from a systematic comparison between the transient data and a mathematical model of the heat conduction in the body of interest.

The purpose of this paper is to introduce numerically efficient solutions for the transient heat transfer in flux-base fins. The contributions of this paper are: first, a unified presentation of transient-fin solutions for three different tip conditions; second, improvement of convergence of the series solutions; and third, for the insulated-tip case, introduction of a closed-form quasi-steady solution. The usefulness of the quasi-steady solution is demonstrated by a comparison with experimental data. The paper is divided into sections on the exact transient solution, improvement of series convergence, the quasi-steady solution, an experimental example, and conclusions.

\section{Exact solutions for flux-base fins}

Traditional fin analysis describes a long, thin, high-conductivity body with a specified temperature on one end and a convection condition over the surface. The temperature distribution in the fin depends on the competing effects of conduction along the fin and convection from the surface of the fin. 
In this section, we present solutions for the transient temperature in flux-base fins. Consider a straight fin initially in equilibrium with the surrounding fluid environment at temperature $T_{e}$. The fin has a constant cross-sectional area, but may be of any shape (pin, rectangular, etc.). For time $t>0$ a steady heat flux is applied to the base of the fin. The temperature in the fin satisfies the following equations

$$
\begin{aligned}
& \frac{\partial^{2} T}{\partial x^{2}}-m^{2}\left(T-T_{e}\right)=\frac{1}{\alpha} \frac{\partial T}{\partial t} ; \quad 0<x<L \\
& \text { at } t=0, \quad T(x, 0)-T_{e}=0 \\
& \text { at } x=0, \quad-k \frac{\partial T}{\partial x}=q_{o} \\
& \text { at } x=L, \quad k_{2} \frac{\partial T}{\partial x}+h_{2}\left(T-T_{e}\right)=0
\end{aligned}
$$

Quantity $m$ is the fin parameter given by $m=\left(h A_{h} / k V\right)^{1 / 2}$. The boundary condition at $x=L$ is a general condition that represents one of three different tip conditions for the fin. For a tip condition of the first kind, setting $k_{2}=0$ and $h_{2}=1$ represents a specified end temperature (at $T=T_{e}$ ). For a tip condition of the second kind, setting $k_{2}=k$ and $h_{2}=0$ represents an insulated-end condition. For a tip condition of the third kind, setting $k_{2}=k$ represents convection at $x=L$. It is not necessary that the convective coefficient at the end of the fin be the same as that along the sides of the fin (i.e. $h_{2} \neq$ $h$ in general), but often $h_{2}=h$ is used.

The details of the unified solution, for all tip conditions, are given in Appendix A. Here, the results will be written out for three tip conditions. For the temperature-end condition (first kind),

$$
T(x, t)-T_{e}=2 \frac{q_{o} L}{k} \sum_{n=1}^{\infty} \frac{\cos \left(\beta_{n} x / L\right)}{m^{2} L^{2}+\beta_{n}^{2}} \times\left[1-\exp \left[-\left(m^{2} L^{2}+\beta_{n}^{2}\right) \alpha t / L^{2}\right]\right]
$$

where $\beta_{n}=(n-1 / 2) \pi$;

for the insulated-end condition (second kind),

$$
\begin{aligned}
T(x, t)-T_{e}= & \frac{q_{o} L}{k} \frac{\left(1-\mathrm{e}^{-m^{2} \alpha t}\right)}{m^{2} L^{2}} \\
& +2 \frac{q_{o} L}{k} \sum_{n=1}^{\infty} \frac{\cos \left(\beta_{n} x / L\right)}{m^{2} L^{2}+\beta_{n}^{2}}\left[1-\exp \left[-\left(m^{2} L^{2}+\beta_{n}^{2}\right) \alpha t / L^{2}\right]\right]
\end{aligned}
$$

where $\beta_{n}=n \pi$;

and, for the convection-end condition (third kind),

$$
\begin{aligned}
T(x, t)-T_{e}= & 2 \frac{q_{0} L}{k} \sum_{n=1}^{\infty}\left(\frac{\beta_{n}^{2}+B_{2}^{2}}{\beta_{n}^{2}+B_{2}^{2}+B_{2}}\right) \frac{\cos \left(\beta_{n} x / L\right)}{m^{2} L^{2}+\beta_{n}^{2}} \\
& \times\left[1-\exp \left[-\left(m^{2} L^{2}+\beta_{n}^{2}\right) \alpha t / L^{2}\right]\right]
\end{aligned}
$$

where $\beta$ satisfies $\beta_{n} \tan \beta_{n}=B_{2}$

and where $B_{2}=h_{2} L / k$.

Each solution contains a series that should be considered in two parts: a transient part with an exponential factor; and, a steady part with no exponential factor. Each of the transient series contains an exponential factor with argument $\left(m^{2} L^{2}+\beta_{n}^{2}\right) \alpha t / L^{2}$, which defines the rate of decay of the transient. The decay rate depends on fin effects (through $m^{2} L^{2}$ ) and also on the tip condition (through $\beta_{n}^{2}$ ).

The steady part of the series converges slowly in each case, on the order of $1 /\left(n^{2} \pi^{2}\right)$. Many terms of the series must be evaluated for accurate numerical values, requiring potentially long computer-evaluation times. In the next section, the convergence speed of the exact solution is improved by replacing the steady series with a fully summed form.

\section{Improvement of series convergence}

It has long been known that classic solutions for the temperature in a body heated on a boundary contain a slowly converging steady-state series [30]. In this section, the convergence of the transient solution is improved by replacing the steady series by a fully summed form. Although the steady-fin solutions are well known, a unified solution is presented with the method of Green's functions.

The steady temperature satisfies the following equations:

$$
\begin{gathered}
\frac{\partial^{2} T}{\partial x^{2}}-\frac{h A_{h}}{k V}\left(T-T_{e}\right)=0 ; \quad 0<x<L \\
\text { at } x=0,-k \frac{\partial T}{\partial x}=q_{o} \\
\text { at } x=L, k_{2} \frac{\partial T}{\partial x}+h_{2}\left(T-T_{e}\right)=0
\end{gathered}
$$

Again, the boundary condition at $x=L$ represents three kinds of tip conditions. Using the method of Green's functions, the steadyfin temperature has the form [31]

$T(x)-T_{e}=\frac{q_{o}}{k} G_{X 2 J}\left(x, x^{\prime}=0\right)$

The symbol for Green's function $G_{X 2 J}$ denotes a Cartesian coordinate system (symbol $X)$, boundary of the second kind at $x=0$ (symbol 2), and boundary of type $J$ at $x=L$ (symbol $J$ ) for $J=1,2$, or 3. This numbering system is used to catalog the many GF available on the GF Library web site [32].

Green's function $G_{X 2 J}$ for the steady-fin is given by

$$
\begin{aligned}
G_{X 2 J}\left(x, x^{\prime}\right)= & R\left(\mathrm{e}^{-m\left(2 L-\left|x-x^{\prime}\right|\right)}+\mathrm{e}^{-m\left(2 L-x-x^{\prime}\right)}\right) / D \\
& +\left(\mathrm{e}^{-m\left|x-x^{\prime}\right|}+\mathrm{e}^{-m\left(x+x^{\prime}\right)}\right) / D
\end{aligned}
$$

where $D=2 m\left(1-R \cdot \mathrm{e}^{-2 m L}\right)$.

Coefficient $R$ is determined by the tip condition:

$R= \begin{cases}-1, & \text { type } 1 \text { at } x=L \\ 1, & \text { type } 2 \text { at } x=L \\ \frac{m L-B_{2}}{m L+B_{2}}, & \text { type } 3 \text { at } x=L\end{cases}$

and where $B_{2}=h_{2} L / k$.

The above GF may be evaluated at $x^{\prime}=0$ and substituted into the above temperature expression, Equation (11), to give

$T(x, t)-T_{e}=\frac{q_{0} L}{k} \frac{\left(\mathrm{e}^{-m x}-\mathrm{e}^{-m(2 L-x)}\right)}{m L\left(1+\mathrm{e}^{-2 m L}\right)}$

where coefficient $R$ is given above.

Alternately, steady-fin solutions may be obtained from computer program TFIN described previously [31] that produces analytical expressions for the steady temperature in fins under a variety of boundary conditions. Program TFIN is also available for download at the Green's Function Library [32].

Next the closed-form steady solutions given above are used in the transient-fin solutions given earlier to replace the slowly converging series. The improved-convergence form of the transient temperature in flux-base fins are given by: for the temperature tip condition (first kind),

$$
\begin{aligned}
T(x, t)-T_{e}= & \frac{q_{o} L}{k} \frac{\left(\mathrm{e}^{-m x}-\mathrm{e}^{-m(2 L-x)}\right)}{m L\left(1+\mathrm{e}^{-2 m L}\right)} \\
& -2 \frac{q_{o} L}{k} \sum_{n=1}^{\infty} \frac{\cos \left(\beta_{n} x / L\right)}{m^{2} L^{2}+\beta_{n}^{2}} \exp \left[-\left(m^{2} L^{2}+\beta_{n}^{2}\right) \alpha t / L^{2}\right]
\end{aligned}
$$

where $\beta_{n}=(n-1 / 2) \pi$; 
for insulated-tip condition (second kind),

$$
\begin{aligned}
T(x, t)-T_{e}= & \frac{q_{o} L}{k} \frac{\left(\mathrm{e}^{-m(2 L-x)}+\mathrm{e}^{-m x}\right)}{m L\left(1-\mathrm{e}^{-2 m L}\right)} \\
& -\frac{q_{o} L}{k} \frac{\mathrm{e}^{-m^{2} \alpha t}}{m^{2} L^{2}}-2 \frac{q_{o} L}{k} \sum_{n=1}^{\infty} \frac{\cos \left(\beta_{n} x / L\right)}{m^{2} L^{2}+\beta_{n}^{2}} \\
& \times \exp \left[-\left(m^{2} L^{2}+\beta_{n}^{2}\right) \alpha t / L^{2}\right]
\end{aligned}
$$

where $\beta_{n}=n \pi$;

and, for the convection tip condition (third kind),

$$
\begin{aligned}
T(x, t)-T_{e}= & \frac{q_{o} L}{k} \frac{\left(\frac{m L-B_{2}}{m L+B_{2}} \mathrm{e}^{-m(2 L-x)}+\mathrm{e}^{-m x}\right)}{m L\left(1-\frac{m L-B_{2}}{m L+B_{2}} \mathrm{e}^{-2 m L}\right)} \\
& -2 \frac{q_{0} L}{k} \sum_{n=1}^{\infty}\left(\frac{\beta_{n}^{2}+B_{2}^{2}}{\beta_{n}^{2}+B_{2}^{2}+B_{2}}\right) \frac{\cos \left(\beta_{n} x / L\right)}{m^{2} L^{2}+\beta_{n}^{2}} \\
& \times\left[\exp \left[-\left(m^{2} L^{2}+\beta_{n}^{2}\right) \alpha t / L^{2}\right]\right]
\end{aligned}
$$

where $\beta$ satisfies $\beta_{n} \tan \beta_{n}=B_{2}$

and where $B_{2}=h_{2} L / k$.

It is instructive to examine these three temperature solutions as a group. Each contains a steady term, and each contains a transient series term. However, the insulated-tip solution uniquely contains another term, a non-series transient. The significance of this nonseries transient term will be addressed in the next section.

\section{Quasi-steady solution}

The insulated-tip fin is of interest for our particular application. The exact temperature expression for this case contains three terms (not two): a steady term, a series term, and a non-series transient term. The series contains an exponential factor with argument $\left(m^{2} \alpha t+\beta_{n}^{2} \alpha t / L^{2}\right)$ and the non-series transient contains an exponential factor with smaller argument $\left(m^{2} \alpha t\right)$. By comparing these arguments, it is clear that as time increases the series term will decay more rapidly. This suggests that a quasi-steady solution may be constructed of the form

$$
T^{q}(x, t)=T^{s}(x)+T^{L}(t)
$$

Here, $T^{s}$ is the steady solution and $T^{L}$ is the non-series transient term from Equation (16). Symbol $T^{L}$ is used to denote "lumped-capacitance", because the non-series transient term is identical to the transient portion of a lumped-capacitance model. Mathematically, there is another view of term $T^{L}$. This term arises from that portion of the Green's function associated with the zero eigenvalue, $\beta_{n}=0$, so that $T^{L}$ is the $n=0$ term of the transient series for the fin temperature. In this view, the quasi-steady approximation is akin to the one-term transient solutions in compact bodies charted many years ago by Heisler [33]. Unlike the Heisler solutions, however, for transient fins there is a spatially varying steady-state solution, and because of the zero eigenvalue, term $T^{L}$ is a function of time only.

The quasi-steady solution is an easily computed algebraic expression, containing no infinite series. Based on the above discussion of exponential arguments, the quasi-steady solution should be accurate for later time. The numerical results given in the next section are presented with the following dimensionless variables:

$$
\begin{gathered}
\theta=\frac{T-T_{e}}{q_{o} L / k} ; \quad \xi=x / L ; \quad \tau=\alpha t / L^{2} \\
M=\sqrt{B i}\left(\frac{L}{V / A_{h}}\right) ; \quad B i=\frac{h\left(V / A_{h}\right)}{k}
\end{gathered}
$$

Here, $M$ is the dimensionless fin parameter. With these parameters, the dimensionless quasi-steady temperature is given by

$$
\theta^{q}(\xi, \tau)=\frac{\mathrm{e}^{-2 M} \mathrm{e}^{M \xi}+\mathrm{e}^{-M \xi}}{M\left(1-\mathrm{e}^{-2 M}\right)}-\frac{\mathrm{e}^{-M^{2} \tau}}{M^{2}} .
$$

and the (dimensionless) exact fin temperature from Equation (6) is given by

$$
\theta(\xi, \tau)=\left[\frac{1}{M^{2}}\left(1-\mathrm{e}^{-M^{2} \tau}\right)+2 \sum_{n=1}^{\infty} \frac{1-\exp \left[-\left(M^{2}+n^{2} \pi^{2}\right) \tau\right]}{M^{2}+n^{2} \pi^{2}} \cos (n \pi \xi)\right]
$$

\subsection{Accuracy of quasi-steady solution}

The quasi-steady solution is compared with the exact transient solution to determine the conditions under which the quasi-steady solution is accurate. Figure 1 shows the (dimensionless) temperature versus position for three different times, all for fin parameter $M=1$. This plot shows that the temperature distribution has a similar shape at each time, and the fixed-shape distribution is shifted upward to higher temperatures as time increases. The quasi-steady and exact temperatures agree closely except at early time $(\tau<0.2)$.

Figure 2 shows the (dimensionless) temperature versus time at three different positions on the fin, all for $M=1$. For $\tau<0.2$ the quasi-steady theory overestimates the exact values at $x / L=0$ and underestimates the exact values at $x / L=1.0$. For all locations the agreement improves as time increases.

Figure 3 shows temperature versus time at $x=0$ for $M=0.2$, 1 , and 5 . At $M=5$ the fin transient ends quickly so that this fin reaches steady-state at about $\tau=0.1$. As $M$ decreases the temperature distribution takes longer and longer to reach steady-state. Fin parameter $M$ may be interpreted as a ratio of thermal resistances: specifically, $M^{2}$ is the thermal resistance along the fin length divided by the convective thermal resistance from the surface of the fin. Thus when $M$ is small, the convective thermal resistance from the surface of the fin is large compared to the thermal resistance along the fin, producing a long, slow transient.

Specific values of the percent error in the quasi-steady theory are given in Table 1 for several values of dimensionless time

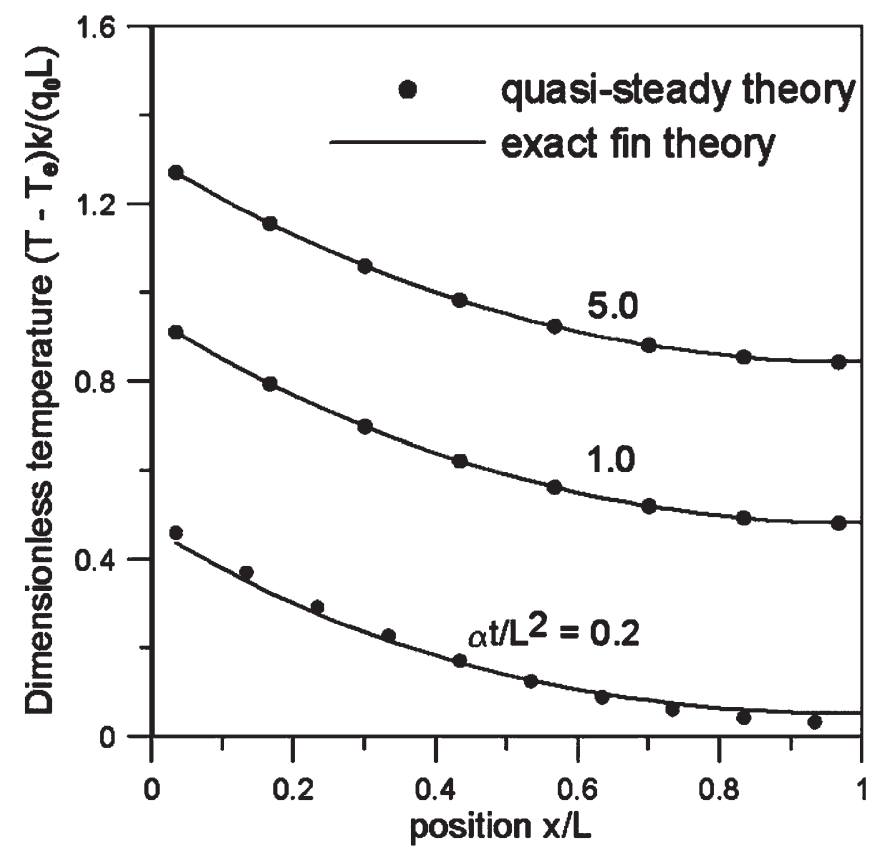

Figure 1. Temperature distribution in a fin of constant cross-section for both quasi-steady theory and exact theory for $M=1$ at dimensionless times $0.2,1.0$, and 5.0. 


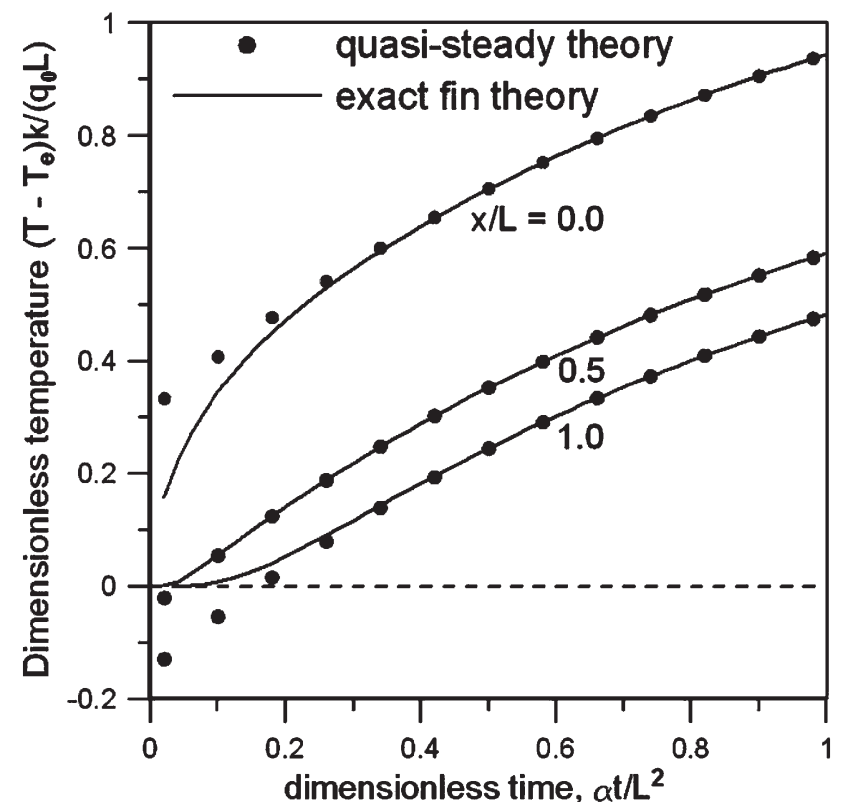

Figure 2. Temperature history in a fin of constant cross-section for both quasi-steady theory and exact theory for $M=1$ at locations $x / L$ $=0.0,0.5$, and 1.0 .

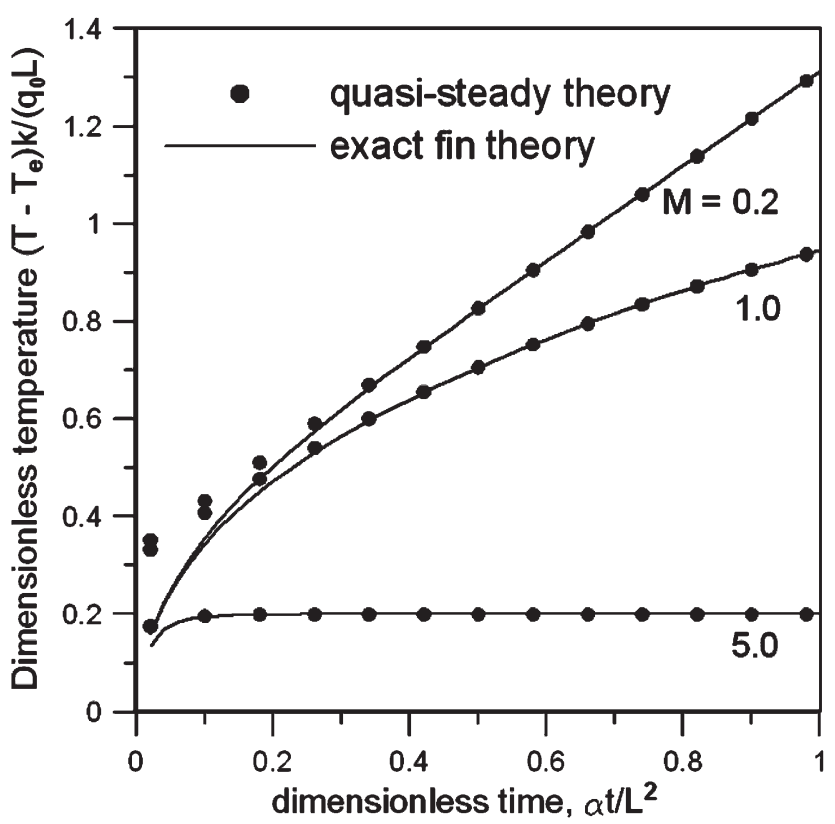

Figure 3. Temperature history in a fin of constant cross-section for both quasi-steady theory and exact theory at location $x / L=0.0$ for $M$ $=0.2,1.0$, and 5.0.

and several values of fin parameter $M$, all at $x / L=1.0$. Table 1 shows that for $M \leq 1$ the error is less than $4 \%$ for dimensionless time $\tau \geq 0.35$, and the error decreases rapidly as time increases. For $M>1$ the region of small error extends to earlier time, for example to $\tau \geq 0.25$ at $M=4$. Error values for other locations on the fin (not shown) are smaller than the Table 1 values and have similar trends. That is, the Table 1 values are worst-case errors, and the guidelines given above provide higher accuracy at locations $x / L<1$.

A comparison of the computer time needed to evaluate numerical values was carried out between the exact series expression and
Table 1. Percent error in quasi-steady temperature evaluated at $x / L=$ 1.0 for several values of time $\tau$ and fin parameter $M$

\begin{tabular}{lrrrrrrr}
\hline$\tau$ & $M=0.1$ & $M=0.2$ & $M=0.5$ & $M=1$ & $M=2$ & $M=3$ & $M=4$ \\
\hline 0.20 & 45.6969 & 45.4860 & 44.0465 & 39.3820 & 26.0504 & 14.0220 & 6.2012 \\
0.25 & 17.0646 & 1.9760 & 16.3713 & 14.4189 & 8.9264 & 4.2077 & 1.4755 \\
0.30 & 7.2802 & 7.2379 & 6.9498 & 6.0235 & 3.4694 & 1.4088 & .3795 \\
0.35 & 3.3684 & 3.3467 & 3.1989 & 2.7262 & 1.4533 & .5010 & .1011 \\
0.40 & 1.6443 & 1.6326 & 1.5532 & 1.3007 & .6388 & .1845 & .0274 \\
0.45 & .8333 & .8268 & .7828 & .6438 & .2900 & .0694 & .0075 \\
0.50 & .4341 & .4304 & .4055 & .3273 & .1347 & .0264 & .0020 \\
1.00 & .0013 & .0012 & .0011 & .0007 & .0001 & .0000 & .0000 \\
2.00 & .0000 & .0000 & .0000 & .0000 & .0000 & .0000 & .0000 \\
\hline
\end{tabular}

the quasi-steady expression. For each expression, the temperature was computed at 300 locations in the range $(0<x / L<1)$, at 300 times in the range $\left(0<\alpha t / L^{2}<1\right)$, and at fin parameter values $M$ $=0.2,1.0$, and 5 , for a total of 270,000 temperature values. For this calculation the exact series expression required $92.8 \mathrm{~s}$ (for convergence within $10^{-6}$ ) and the quasi-steady expression required $2.8 \mathrm{~s}$, less computer usage by a factor of 33 . This calculation was coded in Fortran 77 under the Solaris operating system running on a Sun Blade 2000 with dual $900 \mathrm{MHz}$ processors.

\subsection{Application to parameter estimation}

Next, the use of the quasi-steady expression will be discussed in light of the motivating application for this work, that of determining the heat transfer coefficient from experimental temperature data. This is a parameter estimation problem, in which the theoretical model is used as part of a data analysis procedure. Specifically, the sum-of-square error between the theoretical temperature history and the experimentally measured temperature history is minimized by varying the model parameters (in this case, the heat transfer coefficient). The heat transfer coefficient that minimizes the sum-of-square error is the "best estimate." In the minimization process, the model is evaluated over and over again for different values of the parameters. The computation-intensive nature of the minimization problem was part of our motivation in developing the quasi-steady theory reported here. We were also interested in a model that was easy to implement and easy to understand.

The quality of an estimate depends on the quality of the data, and data quality depends on the sensitivity of the data to the parameter of interest. The sensitivity in this case is the derivative of temperature with respect to the heat transfer coefficient. We have computed the normalized sensitivity in the form

$X=B i \frac{\partial \theta}{\partial B i}$

where $B i$ is the Biot number (dimensionless heat transfer coefficient) and $\theta$ is dimensionless temperature Equation (21). Figure 4 shows this sensitivity at $x / L=0$ plotted versus dimensionless time for three values of fin parameter $M$. Values at other values of $x / L$ (not shown) have similar trends. The important information visible in Figure 4 is that the sensitivity is larger at large dimensionless times, and consequently experimental data at large times is most important for successful parameter estimation. The point of the discussion is that the quasi-steady model, accurate at large dimensionless times, is perfectly suited to estimation of the heat transfer coefficient in fins.

\section{Application to thin cylindrical shell}

The theory for a straight fin of constant cross-section may be extended to include a fin in the form of a thin cylindrical shell. We use this geometry to describe the heat transfer in the outer bear- 


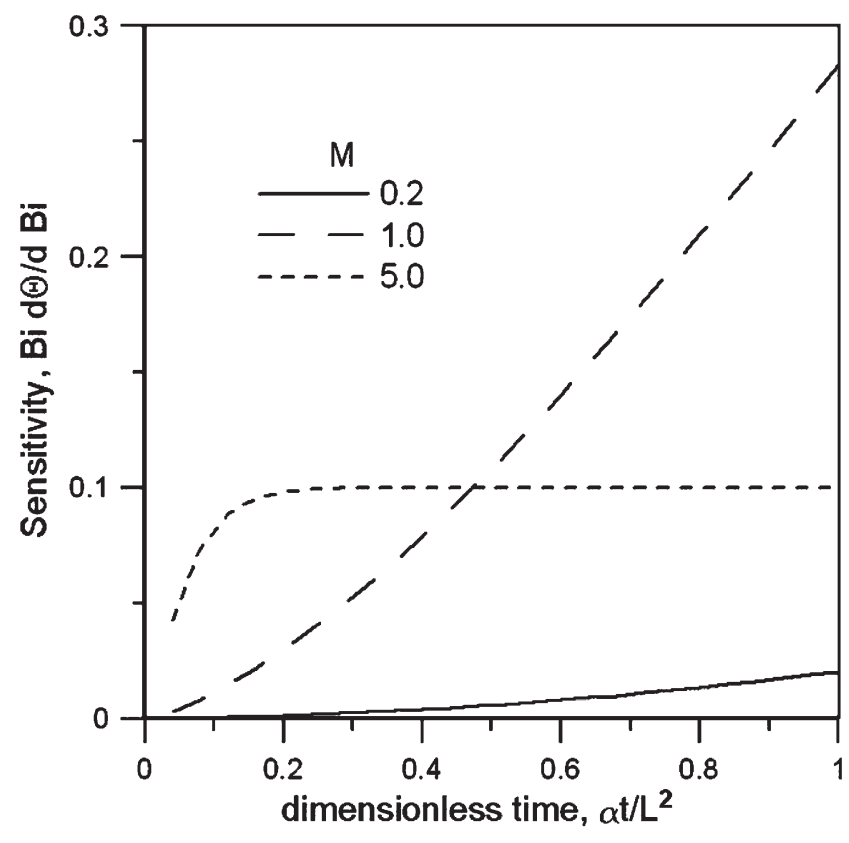

Figure 4. Normalized sensitivity of temperature to Biot number at $x / L$ $=0.0$ for $M=0.2,1.0$ and 5.0. The maximum sensitivity occurs at later time for each case.

ing race of railroad roller bearings, the motivating application for this research.

Consider a cylindrical shell with radius $a$ and thickness $\delta$ for which $\delta \ll a$. The coordinate location on the shell is given by angle $\phi$. For a complete shell the domain is $(0<\phi<2 \pi)$ and for a partial shell the domain is $\left(0<\phi<\phi_{0}\right)$. The transient-fin equation in the cylindrical shell is given by [6, ch. 8]

$\frac{1}{a^{2}} \frac{\partial^{2} T}{\partial \phi^{2}}-\frac{h A_{h}}{k V}\left(T-T_{e}\right)=\frac{1}{\alpha} \frac{\partial T}{\partial t} ; \quad 0<\phi<\phi_{0}$

The thin-shell heat equation may be converted to the straight-fin equation, discussed earlier, by a simple 1:1 mapping. First let $x=$ $\phi L / \phi_{0}$ which maps domain $\left(0<\phi<\phi_{0}\right)$ onto $(0<x<L)$. Then the radius of the thin shell must be related to the fin length by $L=$ $\phi_{0} a$. Now, rearrange these two relationships in the form $\phi=\phi_{0} x /$ $L$ and $a=L / \phi_{0}$ and replace these for $a$ and $\phi$ in the diffusion in Equation (23)

$\frac{1}{a^{2}} \frac{\partial^{2} T}{\partial \phi^{2}}=\frac{1}{L^{2} / \phi_{0}^{2}} \frac{\partial^{2} T}{\partial\left(x^{2} \phi_{0}^{2} / L^{2}\right)}=\frac{\partial^{2} T}{\partial x^{2}}$

With this mapping, the diffusion term for the thin cylindrical shell has been converted into the diffusion term for the straight fin.

\section{Transient heating of a static railroad roller bearing}

This research is part of a larger study to determine the amount of heat needed to explain elevated temperatures that are occasionally observed on the outside of railroad roller bearings. In this section, an experiment is described for transient heating of a non-rotating bearing, and experimental temperatures are compared to the quasi-steady theory.

A railroad class $K$ (6 $1 / 2$ by 12) tapered-roller bearing was used for the non-rotating transient thermal experiment. Heat was supplied to the bearing by two rollers which contained cartridgetype electrical-resistance heaters. Type-K thermocouples were mounted at several locations on the bearing as shown in Figure 5. A large hose clamp was used to fix thermocouples 4 through 11

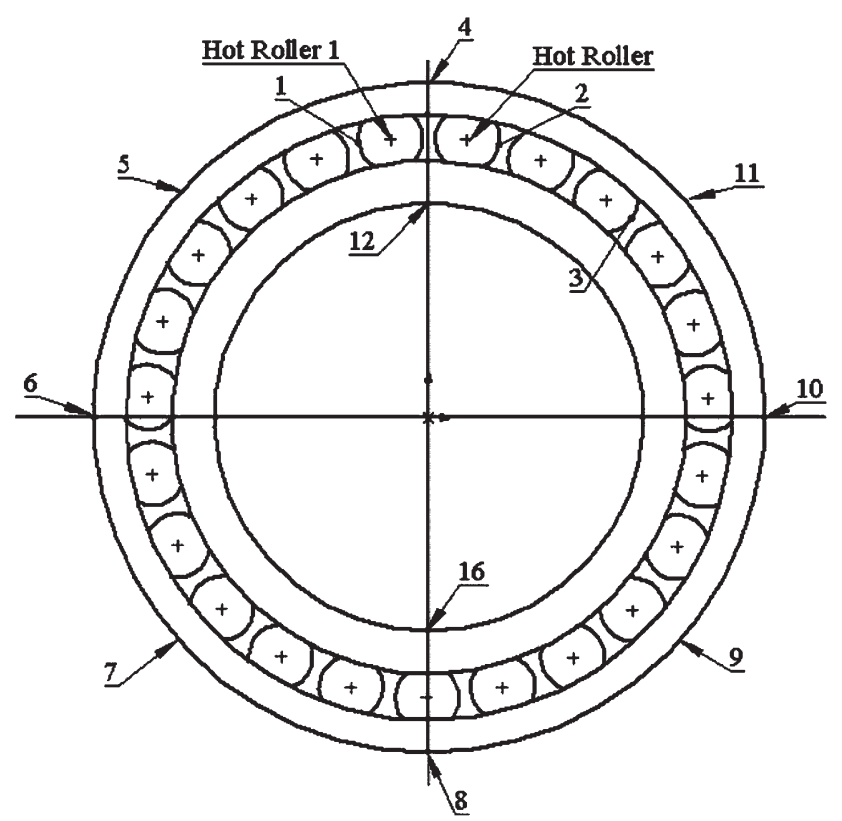

Figure 5. Schematic of the railroad roller bearing used for transient heating tests. Thermocouples 4 through 11 are evenly spaced (every 45 degrees) around the outside of the outer bearing race (cup). The two heated rollers are adjacent to thermocouple 4.

to the outside of the cup (outer bearing race) at the same axial distance from the edge of the cup, corresponding to the middle of the hot rollers. Power was delivered to the two resistance heaters using two variable AC power supplies (variacs). Data acquisition was performed utilizing the Omega Engineering OMB-ChartScan1400 data acquisition system equipped with a 16-channel temperature card. The voltage input to each resistance heater was measured using two CHY 20 multi-meters connected in parallel.

Each experiment was carried out as follows. First, the data acquisition system was initiated, and $120 \mathrm{~s}$ worth of data were acquired and displayed on-screen to ensure that all thermocouples read room temperature. Both variacs were then adjusted to the desired power output with the aid of the digital multi-meters. The voltage and current readings were continuously monitored and recorded every hour to obtain an average power input for each of the two heaters. The data was collected at 1-s intervals, and the data-acquisition software produced a spreadsheet that consisted of 17 columns of data with the first column containing the time stamp (at 20-s intervals), and the remaining 16 columns containing the temperature data (averaged over 20-s intervals) from the 16 thermocouples, respectively. A more complete discussion of the experimental procedure is given elsewhere [34, 35].

A comparison between experimental data and the model is shown in Figure 6. Experimental thermocouple data are shown for thermocouples 6,7 , and 8 corresponding to locations 90 degrees, 135 degrees, and 180 degrees from the heating location. For the model, the suddenly applied heat was taken to be introduced at $\phi=0$ and location $\phi=180$ degrees was treated as the insulatedend of the fin, by symmetry. Model values used to describe the hardened steel cup are given in Table 3 . The best-fit value of the heat transfer coefficient, $h=27.2 \mathrm{~W} \mathrm{~m}^{-1} \mathrm{~K}^{-1}$, was found by a leastsquare regression between the transient data and the model, carried out with the regression tool from a widely available spreadsheet program. Because the quasi-steady model is not accurate at early time, data for the curve fit was limited to $t>1140 \mathrm{~s}(\tau>0.12)$. Figure 6 shows that the quasi-steady model provides a reasonable fit to the experimental data in this time range. 


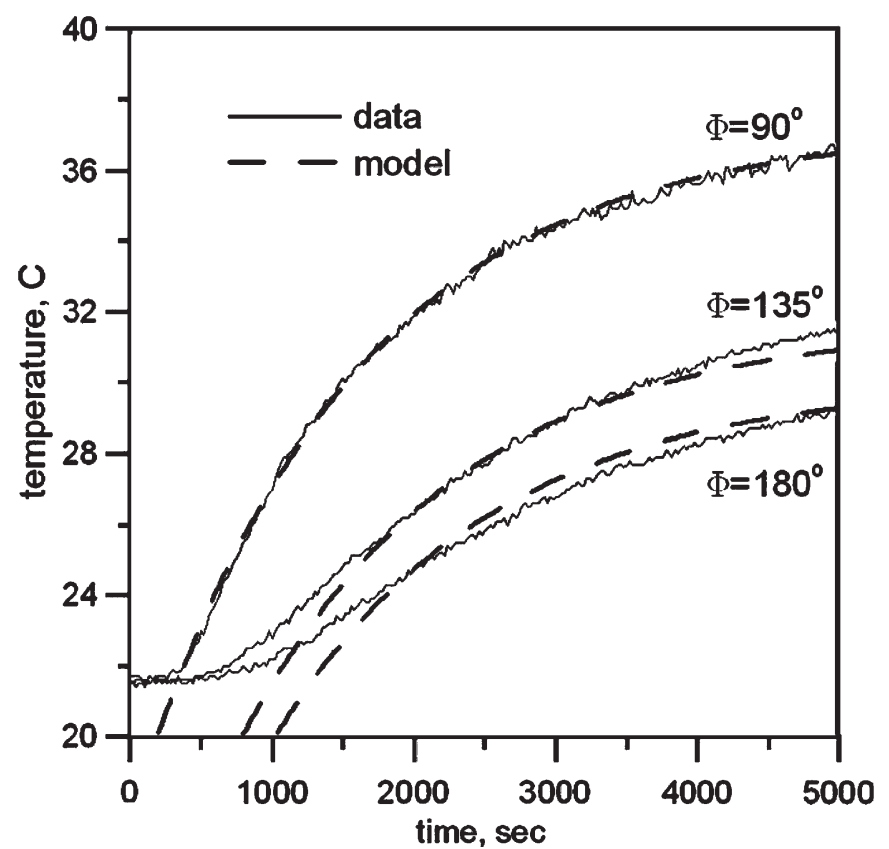

Figure 6. Data from thermocouples 6, 7, and 8 for transient heating of a static bearing, and quasi-steady theory at locations $\phi=90,135$, and 180 degrees. The best-fit value of the heat transfer coefficient is $27.2 \mathrm{~W} \mathrm{~m}^{-2} \mathrm{~K}^{-1}$.

Table 2. Norm and eigenvalues or conditions

\begin{tabular}{lll}
\hline Case & $L / N_{n}$ & $\beta_{n}$ or eigencondition \\
\hline X21 & 2 & $(n-1 / 2) \pi$ \\
X22 & $2 ; n \neq 0$ & $n \pi$ \\
& $1 ; n=0$ & \\
X23 & $2\left[\beta_{n}^{2}+B_{2}^{2}\right] /\left[\beta_{n}^{2}+B_{2}^{2}+B_{2}\right]$ & $\beta_{n} \tan \left(\beta_{n}\right)=B_{2}$
\end{tabular}

Table 3. Parameters for the outer bearing cup used for computing model values

\begin{tabular}{ll}
\hline Parameter & Value \\
\hline$\alpha^{\mathrm{a}}$ & $1.42\left(10^{-5}\right) \mathrm{m}^{2} \mathrm{~s}^{-1}$ \\
$k^{\mathrm{a}}$ & $51.2 \mathrm{~W} / \mathrm{m}^{\mathrm{K}} \mathrm{K}$ \\
$L$ & $0.373 \mathrm{~m}$ \\
$\delta$ & $0.01125 \mathrm{~m}$ \\
$A_{h}$ & $0.1265 \mathrm{~m}^{2}$ \\
$V$ & $0.001469 \mathrm{~m}^{3}$ \\
\hline
\end{tabular}

a Evaluated at $60^{\circ} \mathrm{C}$.

\section{Conclusion}

We have presented a unified theory for transient heat transfer in flux-base fins for three tip conditions. The method may be easily extended to fins with other base conditions. For the particular case of a straight fin with an insulated tip, we have presented a quasi-steady theory in the form of a simple, non-series expression. We expect that the quasi-steady approach could be applied to other transient-fin geometries with an insulated tip, for example the radial fin or other tapered fins for which exact analytical expressions are difficult or unavailable.

A comparison with an exact series solution for the fin transient shows that the quasi-steady theory is accurate within $4 \%$ for dimensionless times greater than 0.35 for small values of the fin parameter $M$. For $M>1$ the accurate range extends to earlier dimensionless times. The accuracy increases for large dimensionless times, where the sensitivity to heat transfer coefficient is largest. The quasi-steady theory is simple and efficient for computing numerical values compared to the exact series solution (33 times faster).

The quasi-steady theory lends itself to repetitive calculations, such as those required for parameter estimation of heat transfer coefficients. Temperatures measured in a transient heating experiment, carried out on a non-rotating railroad roller bearing, were used to find the heat transfer coefficient by a least-square fit comparison with the quasi-steady theory. The results show that the quasi-steady fin model is a simple way to find heat transfer coefficients associated with heat loss from the outside of the bearing. The heat transfer coefficients obtained by this method are intended for future use as an external boundary condition for more elaborate thermal models of this type of bearing.

\section{Appendix A. Transient fin, exact solution}

In this appendix, the series solution for the transient temperature in a flux-base fin is developed by the method of Green's functions.

First, a transformation [36] is used to remove the fin term from the heat conduction equation. Let

$T-T_{e}=W \mathrm{e}^{-m 2 \alpha t}$

and then transform Equations (1)-(4) to give

$\partial^{2} W / \partial x^{2}=\alpha^{-1} \partial W / \partial t ; 0<x<L$

at $t=0, \quad W(x, 0)=0$

at $x=0, \quad-k \partial W / \partial x=q_{0} \mathrm{e}^{m^{2} \alpha t}$

at $x=L, \quad k_{2} \partial W / \partial x+h_{2} W=0$

This transformed problem may be solved by the method of Green's functions in the form [6, p. 165]

$W(x, t)=\frac{\alpha}{k} \int_{t^{\prime}=0}^{t} q_{o} \mathrm{e}^{m^{2} \alpha t^{\prime}} \mathrm{G}\left(x, t \mid x^{\prime}=0, t^{\prime}\right) \mathrm{d} t^{\prime}$

The Green's function associated with function $W$ is that for a plane wall, given by [32]

$G\left(x, t \mid x^{\prime}, t^{\prime}\right)=\frac{X_{0}(0) X_{0}(0)}{N_{0}}+\sum_{n=1}^{\infty} \frac{X_{n}(x) X_{n}\left(x^{\prime}\right)}{N_{n}\left(\beta_{n}\right)} \mathrm{e}^{-\beta_{n}^{2} \alpha\left(t-t^{\prime}\right) / L^{2}}$

The first term (for $n=0$ ) is needed only for a type 2 (insulated) boundary at $x=L$. Eigenfunctions $X_{n^{\prime}}$ eigenvalues $\beta_{n^{\prime}}$ and norm $N_{n}$ are determined by the boundary conditions on the fin. For the flux-base fins of interest here, the eigenfunctions are

$X_{n}\left(\beta_{n}\right)=\cos \left(\beta_{n} x / L\right)$

and the eigenvalues and norms are given in Table 2 . The number system in Table 2 for the three cases listed is X2J where $J=1,2$, or 3 to represent tip conditions of the first kind (temperature), second kind (insulated), or third kind (convection), respectively.

After the time integral in Equation (30) is evaluated, the transformation in Equation (25) can be reversed to find temperature $T$ in the form

$$
\begin{aligned}
T(x, t)-T_{e}= & \frac{q_{o} L}{k} \frac{L}{N_{0}}\left(\frac{1-\mathrm{e}^{-m^{2} \alpha t}}{m^{2} L^{2}}\right)+\frac{q_{o} L}{k} \sum_{n=1}^{\infty} \frac{L}{N_{n}} \\
& \times \frac{\cos \left(\beta_{n} x / L\right)}{\left(m^{2} L^{2}+\beta_{n}^{2}\right)}\left[1-\exp \left[-\left(m^{2} L^{2}+\beta_{n}^{2}\right) \alpha t / L^{2}\right]\right]
\end{aligned}
$$

Again, the first term is only used when the fin tip is insulated (See Kraus et al. [10, p. 765] for an independent derivation of the insulated-tip case). The above expression, with the eigenvalues and norms given in Table 2, is limited to fins with a specified heat flux 
at the base $(x=0)$. However, the same approach could be used for fins with other base conditions with the appropriate plane-wall Green's function. The plane-wall Green's functions for the temperature-base fin (type 1 boundary at $x=0$ ) and the fin with the base temperature applied through a contact conductance (type 3 boundary at $x=0$ ) are available elsewhere (see [6], Appendix $\mathrm{X}$, or [32]).

\section{References}

[1] A. J. Chapman, Transient heat conduction in annular fins of uniform thickness, Chem. Eng. Symp. 55 (29) (1959), pp. 195-201.

[2] A. B. Donaldson and A. R. Shouman, Unsteady-state temperature distribution in a convecting fin of constant area, Appl. Sci. Res. 26 (1-2) (1972), pp. 75-85.

[3] N. V. Suryanarayana, Transient response of straight fins, J. Heat Transf. 97 (1975), pp. 417-423.

[4] N. V. Suryanarayana, Transient response of straight fins part II, J. Heat Transf. 98 (1976), pp. 324-326.

[5] J. Mao and S. Rooke, Transient analysis of extended surfaces with convective tip, Int. Commun. Heat Mass Transf. 21 (1994), pp. 85-94.

[6] J. V. Beck, K. D. Cole, A. Haji-Sheikh and B. Litkouhi, Heat Conduction Using Green's Functions, Hemisphere, New York (1992).

[7] R. H. Kim, The Kantorovich method in the variational formulation to an unsteady heat conduction, Lett. Heat Mass Transf. 3 (1) (1976), pp. 73-80.

[8] A. Aziz and T. Y. Na, Transient response of fins by coordinate perturbation expansion, Int. J. Heat Mass Transf. 23 (1980), pp. 1695-1698.

[9] A. Aziz and A. D. Kraus, Transient heat transfer in extended surfaces, Appl. Mech. Rev. 48 (3) (1995), pp. 317-349.

[10] A. D. Kraus, A. Aziz and J. Welty, Extended Surface Heat Transfer, Wiley, New York (2001).

[11] J. K. Tseng, C. L. Shih and W. J. Wu, Analysis of transient heat transfer in straight fins of various shapes with its base subjected to a decayed exponential function of time in heat flux, Comput. Struct. 47 (2) (1993), pp. 289-297.

[12] C. Y. Cheng and C. K. Chen, Transient response of annular fins of various shapes subjected to constant base heat fluxes, $J$. Phys. D Appl. Phys. 27 (11) (1994), pp. 2302-2306.

[13] Y. Ju, Y. Chou and C. Hsiao, A new approach to the transient conduction in a 2-D rectangular fin, Int. J. Heat Mass Transf. 32 (9) (1989), pp. 1657-1661.

[14] N. Onur, A simplified approach to the transient conduction in a two-dimensional fin, Int. Commun. Heat Mass Transf. 23 (2) (1996), pp. 225-238.

[15] I. V. Singh, K. Sandeep and R. Prakash, Heat transfer analysis of two-dimensional fins using a meshless element free Galerkin method, Numer. Heat Transf. A 44 (1) (2003), pp. 73-84.

[16] A. Campo and A. Salazar, Similarity between unsteady-state conduction in a planar slab for short times and steady-state conduction in a uniform, straight fin, Heat Mass Transf. 31 (5) (1996), pp. 365-370.

[17] A. K. Saha and S. Acharya, Parametric study of unsteady flow and heat transfer in a pin-fin heat exchanger, Int. J. Heat Mass Transf. 46 (20) (2003), pp. 3815-3830.
[18] T. H. Hsu and C. K. Chen, Transient analysis of combined forced and free-convection coduction along a veritcal cicular fin in micropolar fluids, Numer. Heat Transf. A 19 (2) (1991), pp. 177-185.

[19] M. Benmadda and M. Lacroix, Transient natural convection from a finned surface for thermal storage in an enclosure, $\mathrm{Nu}$ mer. Heat Transf. A 29 (1) (1996), pp. 103-114.

[20] D. K. Tafti, L. W. Zhang and G. Wang, Time-dependent calculation procedure for fully developed and developing flow and heat transfer in louvered fin geometries, Numer. Heat Transf. A 35 (3) (1999), pp. 225-249.

[21] A. K. Saha and S. Acharya, Unsteady simulation of turbulent flow and heat transfer in a channel with periodic array of $\mathrm{cu}-$ bic pin-fins, Numer. Heat Transf. A 46 (8) (2004), pp. 731-763.

[22] M. Tutar and A. Akkoca, Numerical analysis of fluid flow and heat transfer characteristics in three-dimensional plate finand-tube heat exchangers, Numer. Heat Transf. A 46 (3) (2004), pp. 301-321.

[23] M. Gharebaghi and I. Sezal, Enhancement of heat transfer in latent heat storage modules with internal fins, Numer. Heat Transf. A 53 (7) (2008), pp. 749-765.

[24] I. Mutlu and T. T. Al-Shemmeri, Steady-state and transient performance of a shrouded longitudinal fin array, Int. Commun. Heat Mass Transf. 20 (1993), pp. 133-143.

[25] G. Stolz Jr., Numerical solution to an inverse problem of heat conduction for simple shapes, J. Heat Transf. 82 (1960), pp. 20-26.

[26] A. M. Osman and J. V. Beck, Investigation of transient heat coefficients in quenching experiments, J. Heat Transf. 112 (1990), pp. 843-848.

[27] I. Dincer, A simple model for estimation of the film coefficients during cooling of certain spherical foodstuffs with water, Int. Commun. Heat Mass Transf. 18 (1991), pp. 431-443.

[28] E. Coment, T. Loulou, and D. Maillet, Estimation of local heat transfer coefficient on a cylinder: comparison between an analytical and an optimization method, Inverse Probl. Sci. Eng. 13 (5) (2005), pp. 449-467.

[29] C. A. Santos, A. Garcia, C. R. Frick, and J. A. Spim, Evaluation of heat transfer coefficients along the secondary cooling zones in the continuous casting of steel billets, Inverse Probl. Sci. Eng. 14 (6) (2006), pp. 687-700.

[30] H. S. Carslaw and J. C. Jaeger, Conduction of Heat in Solids, Oxford University Press, Oxford, UK (1959) p. 185.

[31] K. D. Cole, Computer programs for temperature in fins and slab bodies with the method of Green's functions, Comput. Appl. Eng. Educ. 12 (3) (2004), pp. 189-197.

[32] K. D. Cole, Green's Function Library. Available at http:// www.greensfunction.unl.edu

[33] M. P. Heisler, Temperature charts for induction and constant temperature heating, Trans. Am. Soc. Mech. Eng. 69 (1947), pp. 227-236.

[34] C. M. Tarawneh, K. D. Cole, B. M. Wilson, and K. J. Friesen, A lumped capacitance model for the transient heating of railroad tapered roller bearings, Proceedings of the 2007 ASEEGSW Annual Conference, March 28-30.

[35] C. M. Tarawneh, K. D. Cole, B. M. Wilson, and F. Alnaimat, Experiments and models for the thermal response of railroad tapered roller bearings, Int. J. Heat Mass Transf. (2008); doi: 10.1016/j.ijheatmasstransfer.2008.05.011

[36] M. N. Ozisik, Heat Conduction, Wiley, New York (1993) p. 89. 